\title{
The application of virtual reality technology to understanding psychosis
}

\author{
Commentary on Valmaggia et al. (2016): using virtual reality to investigate \\ psychological processes and mechanisms associated with the onset and maintenance \\ of psychosis: a systematic review
}

\author{
Charlotte Gayer-Anderson ${ }^{1}$
}

Received: 27 May 2016/Accepted: 20 June 2016/Published online: 11 July 2016

(c) Springer-Verlag Berlin Heidelberg 2016

Oculus Rift, Google Cardboard, Samsung Gear, HTC Vive, all types of virtual reality (VR) headsets are fast becoming recognised household names in an industry primed to be worth an estimated 80 billion dollars within the next 10 years (Goldman Sachs). Not only is this new technology taking the gaming (and other) industries by storm, VR applications are becoming more widely used by researchers and clinicians for the assessment and treatment of mental health problems, pioneered by Daniel Freeman and colleagues, specifically in the context of research on paranoia [1].

The review by Valmaggia et al. published in this journal neatly summarises the research that has been carried out using the VR paradigm to explore the role of stress in the development and maintenance of psychosis, and the psychological processes and mechanisms believed to be involved. The findings from this review paint a fairly consistent picture and suggest that: (1) VR can be used to assess paranoid ideation in those from the general population, as well as those deemed at ultra high risk (UHR) of psychosis and those with a clinical diagnosis of psychosis; (2) these experiences are associated with the previous realtime lived experiences of socio-environmental risk factors associated with psychosis in observational studies (e.g., childhood bullying victimisation, life events, and ethnic

This comment refers to the article available at doi:10.1007/s00127016-1245-0.

Charlotte Gayer-Anderson

charlotte.gayer-anderson@kcl.ac.uk

1 Health Service and Population Research Department, Centre for Epidemiology and Public Health, Institute of Psychiatry, Psychology and Neuroscience, King's College London, De Crespigny Park, London SE5 8AF, UK discrimination); (3) these experiences are associated with pre-existing psychological and cognitive attributes (e.g., self-esteem, interpersonal sensitivity, and negative affect); and (4) the VR environment can be finely manipulated to expose participants to different virtual social risk environments (e.g., population and ethnic density) to more precisely ascertain aspects of an environment which pose greater vulnerability to developing psychotic symptoms.

As Valmaggia et al. highlight, the majority of research in this area has used subjective and retrospective self-report questionnaires and/or interviews to measure experiences of stress and adversity. One exception to this is the use of experience sampling methods (ESM), which allow for the measurement of minor stressors in daily life and exacerbation of psychotic symptoms amongst patients with psychosis, their first-degree relatives, and individuals from the general population [2]. However, even this method comes with caveats that VR paradigms can potentially overcome: for example, there are many contextual factors that influence the psychological consequences of daily social environments and this can pose significant challenges in measuring exposure objectively. By contrast, virtual environments can be manipulated to deliberately induce increasing levels of stress; most commonly studies investigating paranoid ideations have used a fairly neutral environment (e.g., an underground train ride, or library) populated by virtual characters (avatars) who do very little other than display occasional potentially ambiguous behaviour (e.g. looking, smiling, talking). Thus, the underlying premise of these studies is to measure intrinsic paranoia in the absence of provocative or stressful situations (i.e., misinterpretations of ambiguous behaviour), and to determine whether these experiences are associated with the occurrence of preceding stressful events or experiences, and/or various cognitive and psychological processes. 
More recently, Veling and colleagues [3] have sought to manipulate the degree of exposure to social stress within the VR paradigm, for example, by varying levels of population density (6 and 20 avatars in the low-stress and highstress conditions, respectively), ethnic density (in the lowstress condition, $80 \%$ of the avatars had the same or similar ethnic appearance, and in the high-stress condition, $20 \%$ had the same ethnic appearance), and hostility (avatars had a neutral expression when the participant approached in the low-stress condition, and an angry and hostile facial expression in the high-stress condition). The authors found that environmental social stress induced paranoia in a dose-response fashion amongst all groups (patients with psychosis, UHR psychosis individuals, siblings of patients with psychosis, and controls with no firstdegree relative with psychosis), and that psychosis liability and negative affect exacerbated the effect of social stress on levels of paranoia.

Importantly, and particularly for the typical measurement of low-level paranoia in those without a clinical disorder, there remains the possibility that these manifestations of increased social withdrawal and anxiety, as well as suspiciousness, constitute rational and adaptive responses to environmental stressors [4]. In other words, whilst those with a psychotic disorder or with clinically relevant symptoms of psychosis may exhibit paranoid beliefs that are delusional and clearly inconsistent with reality, individuals from the general population who rate positively on the self-report questionnaires (or even interview measures) of psychotic experiences may, in fact, be legitimately responding to genuine fears for their safety in the context of exposure to real-world environmental stress or social stress (such as from discriminatory encounters with others, indirect experiences of high-level neighbourhood crime, or direct incidents of violent and intrusive physical threats to the self, etc.). It may then be that standard and typical measures of subclinical paranoia and distress cannot easily distinguish between what may be genuine and rational suspiciousness given an individual's exposure to stressful events and adversity, and paranoia that lies further along the continuum and which is irrational and indicative of a higher susceptibility to developing a psychotic disorder. Whilst questionnaire measures have more recently been devised to assess the multidimensional 'hierarchy' of paranoia [e.g. 5, 6], the VR paradigm offers an experimental method which may be able to discriminate more precisely between the two ends of the spectrum by measuring, following exposure to neutral situations, participants' unfounded paranoid responses to non-intimidating or unthreatening stimuli.

One possible route (of many) of the future of the use of VR methodology in psychosis research is the assessment of predictors of low-level suspiciousness and paranoid beliefs amongst children and adolescents. The rationale for such investigations is several-fold: first, particularly high reports of psychotic symptoms, and specifically paranoid beliefs about others, have been observed amongst adolescents, with prevalence estimates ranging between $10 \%$ to as high as $45 \%$ [7, 8] (higher than what has been found in adults). Importantly, for intervention and preventive strategies, the occurrence and persistence of low-level (sub-threshold) psychotic experiences in this group confer increased risk for not only psychotic disorders later in life [9], but also to other serious mental health problems, such as suicidal ideation [10]. Thus, this is a critical developmental period to explore further to assist in designing targeted interventions that would prevent the emergence of psychosis and other mental health problems.

A second route concerns adolescence, which is characterised by profound social and psychological changes, with naturally heightened sensitivity to experiences of acceptance and rejection by the peer group, and, therefore, to increased feelings of vulnerability. Moreover, exposure to verbal or physical bullying and other forms of social adversity (commonplace amongst youths, with 1 in 5 young people reporting severe maltreatment and abuse at home, in school, and in the community according to a recent UK national survey [11]), no doubt legitimately exacerbate these anxieties. The high proportion of adolescents describing suspiciousness and paranoid beliefs, assessed mainly by means of self-report questionnaires, may, therefore, in part reflect the measurement of warranted hypervigilance to others' behaviour and embody more adaptive concerns relating to theirs and others' social evaluation, often consequential to being exposed to threatening and/or violent acts of aggression [8]. The possibility that positive responses to questions of paranoia and suspiciousness are, in fact, grounded in reality, rather than being pathological symptoms of psychosis, cannot currently be excluded.

On average, three children in every classroom $(\sim 10 \%)$ in the UK have a diagnosable mental disorder, yet $70 \%$ of these have not had appropriate interventions. Simple and effective methods for identifying those with clinically relevant paranoia, and investigation of psychosocial risk factors that are associated with these (e.g., parental separation, socioeconomic disadvantage, abuse, and bullying), and the protective factors that may be providing resilience in those we would expect to stand higher up the paranoia 'hierarchy' (e.g., due to genetic susceptibility, or excessive exposure to psychosocial adversity), are imperative to develop more concrete intervention strategies. Utilising this pioneering VR methodology is a practical and conceivable method to overcome the problems described above, and is an essential next step to acquiring a better comprehension of the determinants and mechanistic 
pathways for low-level psychotic symptoms, and address important questions about the role of risk and resilience in the development of psychotic experiences through adolescence and beyond.

Acknowledgments With thanks to Prof. Craig Morgan who gave constructive comments on the first draft of this commentary.

\section{Compliance with ethical standards}

Conflict of interest None.

\section{References}

1. Freeman D (2008) Studying and treating schizophrenia using virtual reality: a new paradigm. Schizophr Bull 34:605-610

2. Myin-Germeys I, van Os J, Schwartz JE, Stone AA, Delespaul PA (2001) Emotional reactivity to daily life stress in psychosis. Arch Gen Psychiatry 58:1137-1144

3. Veling W, Pot-Kolder R, Counotte J, van Os J, van der Gaag M (2016) Environmental social stress, paranoia and psychosis liability: a virtual reality study. Schizophr Bull. doi:10.1093/schbul/ sbw031

4. Wilson C, Smith ME, Thompson E, Demro C, Kline E, Bussell K, Pitts SC, DeVylder J, Reeves G, Schiffman J (2016) Context matters: the impact of neighborhood crime and paranoid symptoms on psychosis risk assessment. Schizophr Res 171:56-61

5. Freeman D, Garety PA, Bebbington PE, Smith B, Rollinson R, Fowler D, Kuipers E, Ray K, Dunn G (2005) Psychological investigation of the structure of paranoia in a non-clinical population. Br J Psychiatry 186:427-435

6. Green CEL, Freeman D, Kuipers E, Bebbington PE, Fowler D, Dunn G, Garety PA (2008) Measuring ideas of persecution and social reference: the Green et al. paranoid thought scales (GPTS). Psych Med 38:101-111

7. Laurens KR, Hobbs MJ, Sunderland M, Green MJ, Mould GL (2012) Psychotic-like experiences in a community sample of 8000 children aged 9-11 years: an item response theory analysis. Psych Med 42:1495-1506

8. Sousa M, Carvalho CB, da Motta C, Cabral J, Pereira V, Caldeira SN, Peixoto E (2015) Characterization and predictors of paranoid ideation in youths. Int J Psychol Behav Sci 9(2):1710-1717

9. Poulton R, Caspi A, Moffitt TE, Cannon M, Murray R, Harrington H (2000) Children's self-reported psychotic symptoms and adult schizophreniform disorder: a 15-year longitudinal study. Arch Gen Psychiatry 57:1053-1058

10. Kelleher I, Cederlöf M, Lichtenstein P (2014) Psychotic experiences as a predictor of the natural course of suicidal ideation: a Swedish cohort study. World Psychiatry 13:184-188

11. Radford L, Corral S, Bradley C, Fisher HL, Bassett C, Howat N, Collishaw S (2011) Child abuse and neglect in the UK today. National Society for the Prevention Against Cruelty to Children, London 